\title{
FORMATION FLYING SATELLITE CONTROL AROUND THE L2 SUN-EARTH LIBRATION POINT
}

\author{
By \\ Nicholas H. Hamilton \\ United States Air Force \\ and \\ David Folta and Russell Carpenter \\ NASA/GSFC
}

\begin{abstract}
This paper discusses the development of a linear control algorithm for formations in the vicinity of the L2 sun-Earth libration point. The development of a simplified extended Kalman filter is included as well. Simulations are created for the analysis of the stationkeeping and various formation maneuvers of the Stellar Imager mission. The simulations provide tracking error, estimation error, and control effort results. For formation maneuvering, the formation spacecraft track to within 4 meters of their desired position and within 1.5 millimeters per second of their desired zero velocity. The filter, with few exceptions, keeps the estimation errors within their three-sigma values. Without noise, the controller performs extremely well, with the formation spacecraft tracking to within several micrometers. Each spacecraft uses around 1 to 2 grams of propellant per maneuver, depending on the circumstances.
\end{abstract}

\section{INTRODUCTION}

NASA would like to fly many distributed spacecraft missions in the next decade and beyond. Among these concepts, the Magnetospheric Multiscale and Magnetospheric Constellation ${ }^{1}$ will study the magnetotail of the earth, Stellar Imager ${ }^{2}$ (SI), MAXIM ${ }^{3}$, and Constellation- $\mathrm{X}^{4}$ will image stars and black holes respectively, and the Laser Interferometer Space Antenna $^{5}$ (LISA) will attempt to detect gravitational waves. Some of these missions, including SI and MAXIM, plan to operate near the Sun-Earth libration points, and have very precise formation control requirements.

Farquhar ${ }^{6}$ initially examined control techniques for the station-keeping of satellites orbiting a libration point. Gomez, Masdemont, and $\mathrm{Simo}^{7}$ have extensively studied dynamics and control problems involving libration points. Scheeres and $\mathrm{Vinh}^{8}$ have researched the dynamics and control of relative motion of two spacecraft in unstable orbits. Hoffman ${ }^{9}$ and $\mathrm{Wie}^{10}$ used the linear quadratic regulator to control problems involving the Earth-Moon collinear libration points. Currently, diverse approaches to support these mission types are under study. In this paper, the use of high fidelity dynamics along with a discrete linear- quadratic-regulator framework is applied for control of SI in the vicinity of the L2 Sun-Earth libration point. The development of a simplified extended Kalman filter is included as well.

\section{SI MISSION DESIGN AND CONTROL REQUIREMENTS}

SI is a concept for a space-based, UV-optical interferometer, proposed by Carpenter and Schrijver ${ }^{11}$. The purpose of the mission is to view many stars with a sparse aperture telescope in an attempt to better understand the various effects of stars' magnetic fields, the dynamos that generate them, and the internal structures and dynamics of stars. The leading concept for SI is a 500-meter diameter Fizeau-type interferometer composed of 30 small drone satellites that reflect incoming light to a hub satellite. The hub will recombine, process, and transmit the information back to Earth. As Figure 1 shows, in this concept, the hub satellite lies halfway between the surface of a sphere containing the drones and the sphere origin. Focal lengths of both $0.5 \mathrm{~km}$ and $4 \mathrm{~km}$ are being considered. This would make the radius of the sphere either $1 \mathrm{~km}$ or $8 \mathrm{~km}$. Details of the required formation geometry may be found in the appendix.

The type of orbit and location in space is an important part of mission design. The best orbit choice 
for the formation after consideration of gravity gradients, scattered and stray light, and element replacement is a Lissajous orbit around the Sun-Earth L2 point. The y-amplitude of the Lissajous orbit will be about $600,000 \mathrm{~km}$, but is not critical to the mission. With this orbit, SI will be able to cover the entire sky every half year while maintaining an aim perpendicular to the sun. For useful imaging, SI must aim within 10 degrees of perpendicular from the sun.

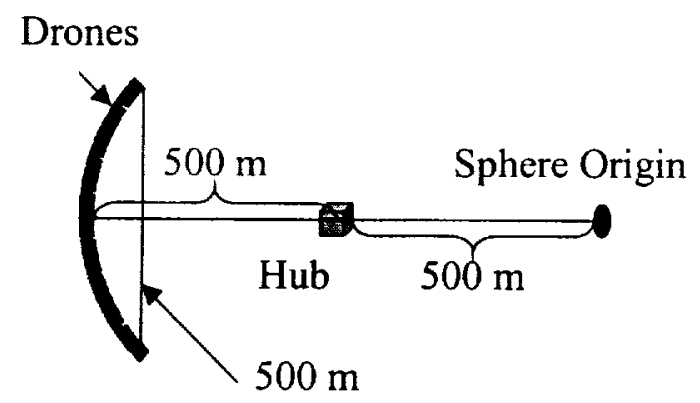

Figure 1. Satellite formation with focal length $500 \mathrm{~m}$

To function properly, SI will need to accommodate a wide range of control functions. In addition to maintaining its desired trajectory around $\mathrm{L} 2$, the formation must slew about the sky requiring movement of a few kilometers and attitude adjustments of up to 180 degrees. While imaging, though, the drones must maintain position within 3 nanometers of accuracy in the direction radial from the hub and within 0.5 millimeters of accuracy along the sphere surface. The accuracy required for attitude control while imaging is 5 milli-arcseconds tip and tilt (rotations out of the surface of the sphere). The rotation about the axis radial from the hub (rotation within the sphere) is a much less stringent 10 degrees.

To achieve these requirements, Leitner and Schnurr ${ }^{12}$ propose a three-tiered formation control approach. The first tier is "rough" control using radio frequency $(\mathrm{RF})$ ranging and modified star trackers for sensors and thrusters for actuators. The relative positions will be controlled to within a few centimeters. This level will drive lost-in-space emergencies, formation initialization, large translations due to formation slewing, collision avoidance, and maintenance of the formation's trajectory about L2. The RF ranging system will provide range measurements, and the modified star trackers will provide azimuth and elevation measurements. The actuators for this tier will be four lowthrust, high specific impulse (Isp) thrusters. The thrust level will be on the Newton to milli-Newton order of magnitude. It is this tier that this paper primarily addresses, although these results could be applied at the next tier as well.
The second tier is "intermediate" control with a modulated laser ranging system as sensors and thrusters as actuators. The relative positions will be controlled to within 50 microns at this tier. This level will drive primary attitude adjustments and small translation maneuvers. Twelve 10-100 micro-Newton Indium Field Emission Electric Propulsion (FEEP) thrusters will be used for this level. This propulsion technology is currently available, but by 2015 should be vastly improved. Basically, this tier functions to smooth the transition from the rough control of the first-tier to the fine precision control of the third-tier.

The third-tier is fine precision control. At this level, the satellites themselves will not move; instead, the optics will be adjusted by extremely accurate mechanical devices with an accuracy in the nanometer range. Rather than having a traditional sensor to determine measurements, phase diversity and wave-front error (WFE) sensing algorithms, using data from the incoming light rays, will determine the needed control. Currently, phase diversity and WFE sensing are in their infancy for use with spacecraft and formation flying concepts.

\section{Controller Development}

A common approximation in research of this type models the dynamics of a satellite in the vicinity of the sun-earth L2 point using the circular restricted three-body assumptions. These assumptions only account for gravitational forces from the sun and Earth. The moon is also included, but not as an independent body. The masses of the earth and moon are combined and assumed to be at the earth-moon barycenter. The motion of the sun and the earth-moon barycenter is also assumed to be circular around the system barycenter.

This analysis uses high fidelity dynamics based on a simulation named Generator that Purdue University has developed. Generator creates much more realistic Lissajous orbits than those derived from the circular restricted three-body problem. Using ephemeris files, Generator takes into account the effects of eccentricity, an independent moon, the other planets of the solar system, and solar radiation pressure. The resulting Lissajous orbit can then be used as a more accurate reference orbit. In addition to providing the reference positions and velocities, Generator also numerically computes and outputs the linearized dynamics matrix, $A$, for a single satellite at each epoch. This data can be used onboard for autonomous computation by simple uploads or onboard computation as a background task of the 36 matrix elements and the state vector. A Generator reference orbit is shown in Figure 2. The origin in Figure 2 is the earth. The $X$ coordinate connects the two primary bodies, the $Z$ coordinate is parallel to their angular velocity of the system, $\omega$, and the $Y$ coordinate completes a right-handed system. The 
reference orbit period is 359 days and the scale is in kilometers.



Figure 3. Generator based 359 day reference orbit around $\mathrm{L}_{2}$

The coordinates of a satellite can be written as

$$
\begin{aligned}
& X=X_{0}+x \\
& Y=Y_{0}+y \\
& Z=Z_{0}+z,
\end{aligned}
$$

where $X_{0}, Y_{0}$, and $Z_{0}$ are the coordinates of any one of the libration points. The linearized equations of motion about a collinear point can be expressed in statespace notation as

$$
\dot{\mathbf{x}}=A \mathbf{x}
$$

where

$$
\mathbf{x}=\left[\begin{array}{llllll}
x & y & z & \dot{x} & \dot{y} & \dot{z}
\end{array}\right]^{T},
$$

and $A$ is output from Generator.

For SI, the overall formation must follow a prescribed path about the libration point, and individual satellites must maintain desired relative configurations; i.e. the controller must be of the tracking type. Define the state error as

$$
\widetilde{\mathbf{x}}(t)=\mathbf{x}(t)-\mathbf{x}_{\text {ref }}(t)
$$

so that the desired linear control law is

$$
\mathbf{u}=-K(t) \widetilde{\mathbf{x}},
$$

The problem is therefore in the form of the linearquadratic-regulator (LQR), which provides the gain $K$ that minimizes the cost function

$$
J=\int_{i_{0}}^{\prime}\left\{\widetilde{\mathbf{x}}^{T}(\tau) W \widetilde{\mathbf{x}}(\tau)+\mathbf{u}^{T}(\tau) V \mathbf{u}(\tau)\right\} d \tau
$$

where $W$ is a penalty on the mean square tracking errors, and $V$ is a penalty on the control energy. Assuming the reference satisfies the state differential equation, the closed loop dynamics are

$$
\dot{\widetilde{\mathbf{x}}}=(A-B K(t)) \widetilde{\mathbf{x}},
$$

where $\mathbf{u}$ is the control vector and $B$ is the matrix that maps the control effort to the state-space. The control is modeled as ideally applied acceleration in the $x, y$, and $z$ directions. Therefore,

$$
B=\left[\begin{array}{lll}
0 & 0 & 0 \\
0 & 0 & 0 \\
0 & 0 & 0 \\
1 & 0 & 0 \\
0 & 1 & 0 \\
0 & 0 & 1
\end{array}\right] \quad \text { and } \mathbf{u}=\left[\begin{array}{l}
u_{1} \\
u_{2} \\
u_{3}
\end{array}\right]
$$

where $u_{1}$ is the control in the $x$ direction, $u_{2}$ is the control in the $y$ direction, and $u_{3}$ is the control in the $z$ direction.

Letting subscript 1 denote the hub, the controller will regulate the hub to follow a desired path around $\mathrm{L} 2$, while the drones, denoted by higher subscripts, will be controlled relative to the hub only. Thus the drone's state vectors are actually relative to the hub, not the libration point, and for satellite 2 , the tracking error obeys

$$
\dot{\mathbf{x}}_{2}=A \widetilde{\mathbf{x}}_{2}+B \mathbf{u}_{2}-B \mathbf{u}_{1} \text {, }
$$

For the complete formation, it is convenient to redefine $A$ and $B$ such that

$$
A=\left[\begin{array}{llll}
A_{1} & & & \\
& A_{2} & & \\
& & \ddots & \\
& & & A_{j}
\end{array}\right], B=\left[\begin{array}{ccccc}
B_{1} & & & & \\
-B_{1} & B_{1} & & & \\
-B_{1} & & B_{i} & & \\
\vdots & & & \ddots & \\
-B_{1} & & & & B_{j}
\end{array}\right]
$$

The system is discretized using a sample-and-hold approach. The state transition matrix is created from the dynamics partials output from Generator by

$$
\Phi\left(t-t_{0}\right)=e^{A\left(t-t_{0}\right)}=\sum_{k=0}^{\infty} \frac{A^{k}\left(t-t_{0}\right)^{k}}{k !},
$$

truncating the series after the quadratic term. Although the $A$ matrix from Generator is slowly time-varying, it is assumed to be constant over the time step, which is in all cases much smaller than the "period" of the Lissajous orbit. At each time step, the steady-state LQR control gain is computed from the solution of the discrete algebraic Riccati equation corresponding to the state transition matrix for that epoch.

Since the measurements are critical to the feasibility of the SI control concept, and since the types of measurements under consideration are nonlinear in the states of the controller described above, a simplified extended Kalman filter that uses the dynamics described above, augmented by zero-mean, white, Gaussian process and measurement noise was also studied. The discretized state dynamics for the filter are 


$$
\widetilde{\mathbf{x}}_{k+1}=A_{d} \widetilde{\mathbf{x}}_{k}+B_{d} \mathbf{u}_{k}+w
$$

where $w$ is the random process noise vector. The (nonlinear) measurement model is

$$
\mathbf{y}_{k}=m\left(\widetilde{\mathbf{x}}_{k}\right)+v
$$

The covariances of process and measurement noise are

$$
E\left[w w^{T}\right]=Q, E\left[v v^{T}\right]=R,
$$

For the hub, we assume Earth-based range, azimuth, and elevation, are measured. To keep the filter simple, the angles were assumed to be measured in relative to the ecliptic plane.

For the drone satellites, positions are determined relative to the hub satellite. Range is a scalar, so whether it comes from a sensor on the hub or the drone is unimportant. Azimuth and elevation, on the other hand, usually come from sensors on the drone spacecraft. They are in the frame of reference of the local coordinate system centered on the drone. Because the states of the drone spacecraft are represented with respect to the hub spacecraft, and the angles are with respect to the drone spacecraft, a coordinate transformation is required. However, if the local coordinate system on the drone is oriented the same as the reference coordinate system on the hub $\left(b_{1}\right.$ is aligned with $x, b_{2}$ is aligned with $y$, and $b_{3}$ is aligned with $z$ ), the position of the drone can be determined from simple trigonometry.

For convention positive azimuth is defined as counter-clockwise from the $b_{2}$ direction in the $b_{1}-b_{2}$ plane. Positive elevation is defined from the $b_{1}-b_{2}$ plane upwards in the positive $b_{3}$ direction. The position of the drone relative to the hub is then

$$
\begin{gathered}
x=r \cos (e l) \sin (a z), \\
z=-r \sin (e l),
\end{gathered}
$$

where $\mathrm{r}$ is the range, $e l$ is the elevation, and $a z$ is the azimuth as shown in Figure 3.

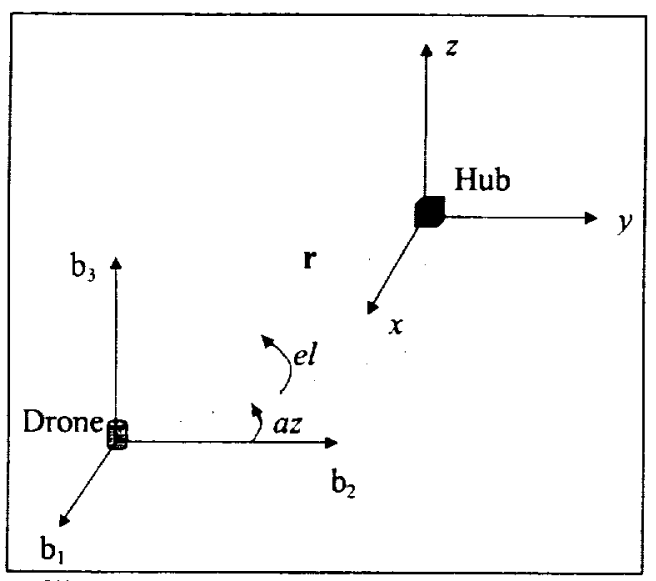

Figure 3. Range, azimuth, and elevation of one spacecraft
In terms of the states, the measurements for one satellite are

$$
r=\sqrt{x^{2}+y^{2}+z^{2}}
$$

$$
e l=\sin ^{-1}\left(\frac{-z}{r}\right), \text { and } a z=\sin ^{-1}\left(\frac{x}{r \cos (e l)}\right)
$$

These models are used with standard techniques, e.g. as may be found in Brown and Hwang ${ }^{14}$, to construct the simplified extended Kalman filter for this study.

\section{RESULTS}

Three different scenarios make up the position control problem-maintaining the Lissajous orbit, slewing the formation to aim at another star, and reconfiguring the formation to take another snapshot of a star when necessary. These three scenarios are treated independently. To determine the amount of fuel, the velocity change, or $\Delta V$, is needed. The $\Delta V$ in each direction is found by numerical integration:

$$
\Delta V_{x}=\sum_{k=1}^{359}\left|u_{x}\right| T, \Delta V_{y}=\sum_{k=1}^{359}\left|u_{y}\right| T, \Delta V_{z}=\sum_{k=1}^{359}\left|u_{z}\right| T
$$

where $T$ is the maneuver interval, the upper limit of the sum is the simulation length. The absolute value of the control is taken because the direction of the maneuver has no bearing on the fuel used. The total $\Delta V$ for one simulation is calculated by

$$
\Delta V=\Delta V_{x}+\Delta V_{y}+\Delta V_{z}
$$

\section{Lissajous Orbit Maintenance}

Following the Lissajous orbit is not a problem of formation control, but rather a problem of maintaining an orbit. Therefore, only the hub satellite needs simulation to determine the amount of control and fuel needed to maintain a Lissajous orbit. The results can be extended similarly to other satellites in the formation.

The continuous state weighting matrix and the continuous control weighting matrix are chosen to be

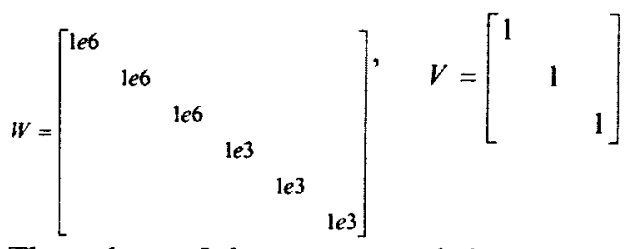

The values of the process and the measurement noise covariance matrix for the hub satellite are chosen to be 


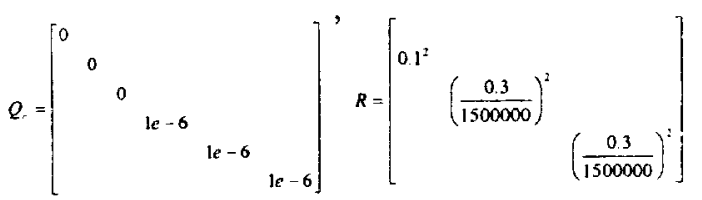

The strength of the process noise is set at a value large enough to be noticed, but not so much as to constrict or destabilize the system. The first term in the measurement noise covariance matrix assumes range measurements from the earth to the hub within $0.1 \mathrm{~km}$ or 100 meters. The second and third terms assume that the arc lengths corresponding to the azimuth and elevation angles are three times less accurate than the range measurements. To find the angular accuracy, simply divide the accuracy of arc length by the range. The $\mathrm{L}_{2}$ point is approximately 1.5 million kilometers from the earth and is used as a roughly constant scaling distance.

The measurement covariance for the drone satellites is different from that of the hub:

$$
R=\left[\begin{array}{lll}
0.0001^{2} & & \\
& \left(\frac{0.0003}{0.5}\right)^{2} & \\
& & \left(\frac{0.0003}{0.5}\right)^{2}
\end{array}\right]
$$

Here, the range measurement is assumed to be accurate to within 0.1 meters, with three times less accurate arc lengths. The range from the hub to the drones is the focal length of the interferometer (either 0.5 or $4 \mathrm{~km}$ ).

The initial covariances for the hub and drones are

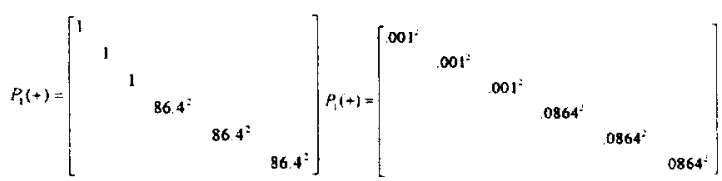

respectively. For the hub, this assumes about $1 \mathrm{~km}$ accuracy for position (10 times greater than measurement noise covariance) and 1 meter per second for velocity. The 86.4 term is the conversion to kilometers per day from meters per second. For drone satellites, the covariance corresponds to a position accuracy of 1 $\mathrm{m}$ and velocity accuracy of 1 millimeter per second Figure 4 shows the tracking errors plotted against time for a simulation one year, with a time step of 1 day. The plots on the left are position tracking errors, and the plots on the right are velocity tracking errors. Running many simulations, it can be seen that the steady-state position tracking errors are within $0.25 \mathrm{~km}$ for each direction, and the steady-state velocity tracking errors are within $7.5 \mathrm{e}-4$ meters per second for each direction. Because the estimation errors are based on randomness, many simulations must be run to determine useful re- sults. Figure 5 shows the estimation errors of a dozen simulations.
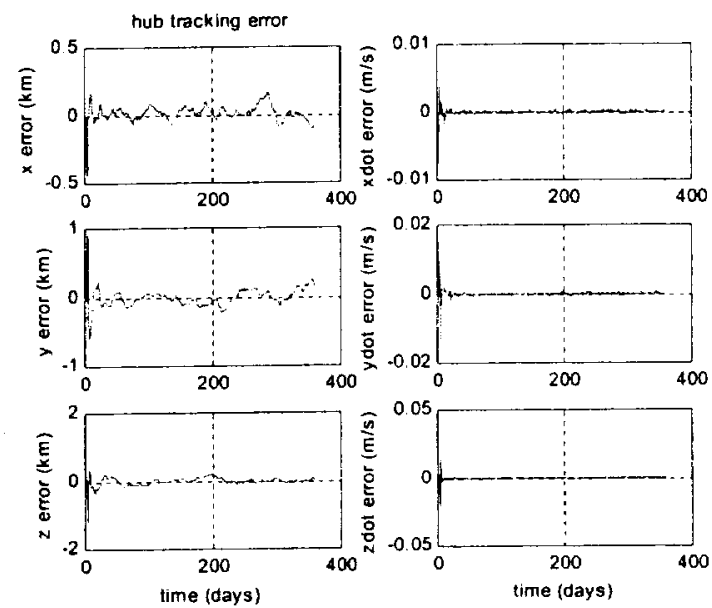

Figure 4. Hub tracking errors

The plots on the left are the position estimation errors, and the plots on the right are the velocity estimation errors. The blue lines represent the actual estimation errors, and the red lines represent the threesigma value of the covariance.
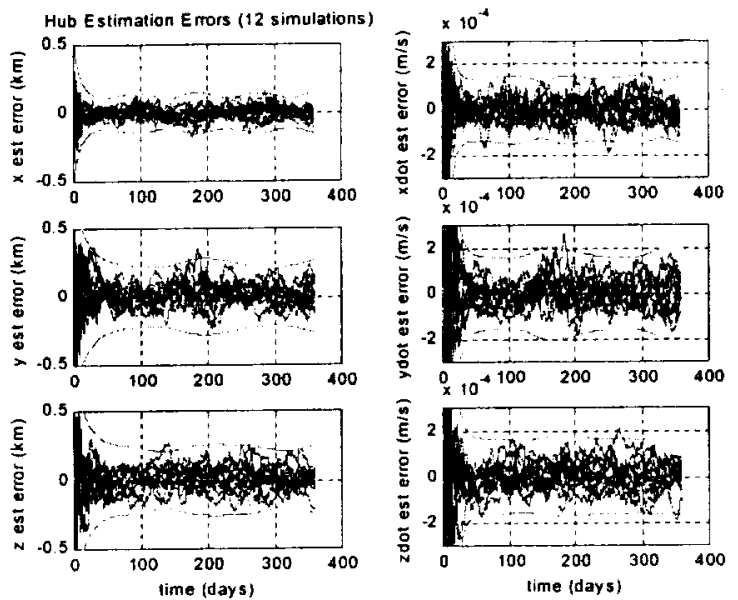

Figure 5. Hub estimation errors (12 simulations)

The estimation errors lie within the threesigma values of the covariance with very few exceptions. The position estimation errors are within 250 meters, and the velocity estimation errors are within $2 \mathrm{e}-4$ meters per second. Figure 6 shows the control effort over the course of one simulation. Averaging the determined $\Delta V$ from a dozen simulations, the $\Delta V$ required to keep a satellite in a Lissajous orbit about L2 for 359 days is approximately 0.38 meters per second. Using the rocket equation, and Stellar Imager of initial spacecraft mass of $550 \mathrm{~kg}$ for the hub, a $100 \mathrm{~kg}$ for a drone, and the Isp for the low-thrust, high-Isp thruster of 10000 seconds, the amount of propellant needed to 
maintain a Lissajous orbit for 359 days is less than 2.2 grams for the hub and less than 0.4 grams for each drone.
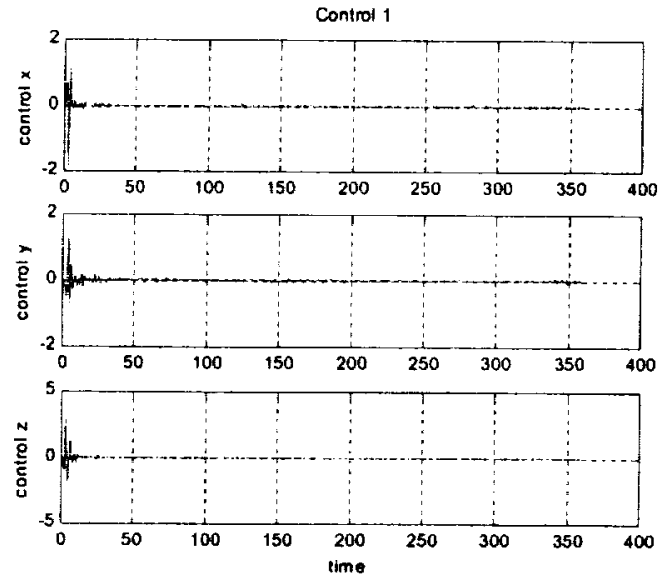

Figure 6. Hub control effort

\section{Formation Slewing}

A key part of the SI mission is to image many stars. Following a Lissajous orbit around L2, SI could view the entire sky approximately every half-year while slewing about just the radial ( $x$ ) axis. This will also maintain the aiming angle perpendicular to the sun. The formation slewing simulation follows a similar algorithm as the Lissajous orbit simulation.

For this simulation, the maneuver interval is 1 minute and the length of the simulation is 24 hours, using a constant $A$-matrix from day 2 of the year-long simulation made above. Various slew angles are examined. All other tuning parameters are the same, except that their discretized values are adjusted by the change in time step. The strength of the process noise is different from the Lissajous orbit simulation,

$$
Q_{c}=\left[\begin{array}{llllll}
0 & & & & \\
& 0 & & & & \\
& 0 & & & \\
& & \mathrm{le}-24 & & \\
& & & \mathrm{le}-24 & \\
& & & & \mathrm{le}-24
\end{array}\right]
$$

As before, the strength of the process noise is set at a value large enough to be noticed, but not so much as to constrict or destabilize the system.

The hub tracking error, estimation error, and control are found for focal lengths of both 0.5 and 4 $\mathrm{km}$. Each drones' tracking error, estimation error, and control are determined as well. For conciseness, only the results for one drone will be shown (satellite 2). Two slewing angles are investigated, 90 degrees and 30 degrees. Figure 7 provides an image of the entire SI formation slewing 90 degrees, with a $0.5 \mathrm{~km}$ focal length.
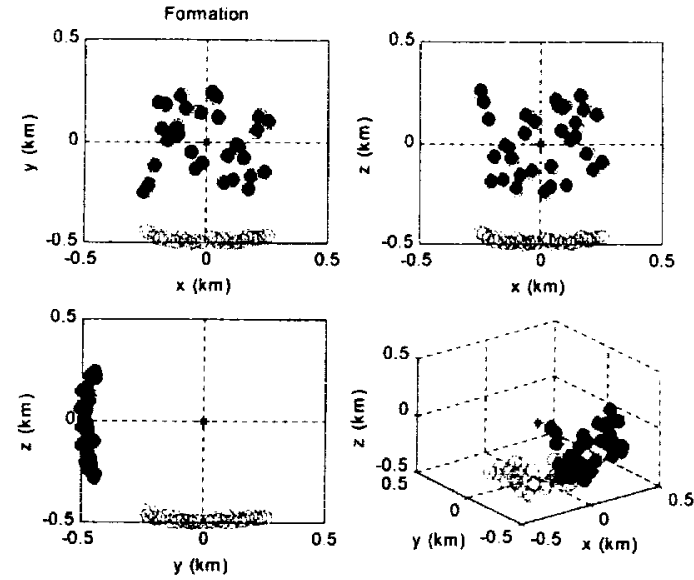

Figure 7. SI formation before and after 90 degree slew ( $0.5 \mathrm{~km}$ focal length)

The black dots represent drones at the beginning of the simulation, and the open circles represent drones at the end of the simulation. The hub is the black asterisk at the origin. The upper-right plot illustrates the Golomb $^{15}$ rectangle formation projected into the $x-z$ plane. The lower-left plot clearly shows the drones slewing 90 degrees about the hub-centered $\mathrm{x}$ axis.

Figures 8 and 9 show the tracking errors for the hub and drone. Although the plots are specific to a 90 degree slew with a $0.5 \mathrm{~km}$ focal length, they are qualitatively representative of all the different slew angle and focal length scenarios.
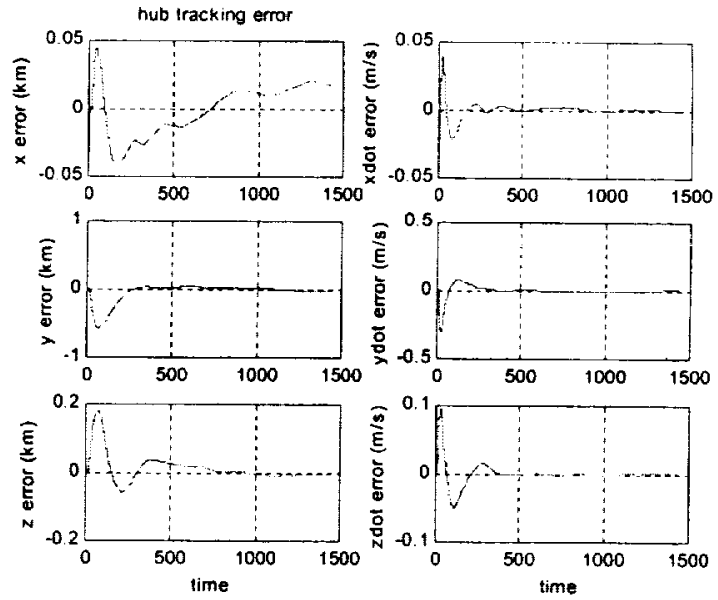

Figure 8. Hub tracking error ( 90 degree slew and 0.5 $\mathrm{km}$ focal length)

For both 90 degree and 30 degree slews with either a 0.5 or $4 \mathrm{~km}$ focal length, the hub tracks to within 50 meters of its reference position and to within 5 millimeters per second of its desired velocity. The drones all track to within 3 meters of their desired reference positions and to within 1 millimeter per second of their desired zero velocities. 

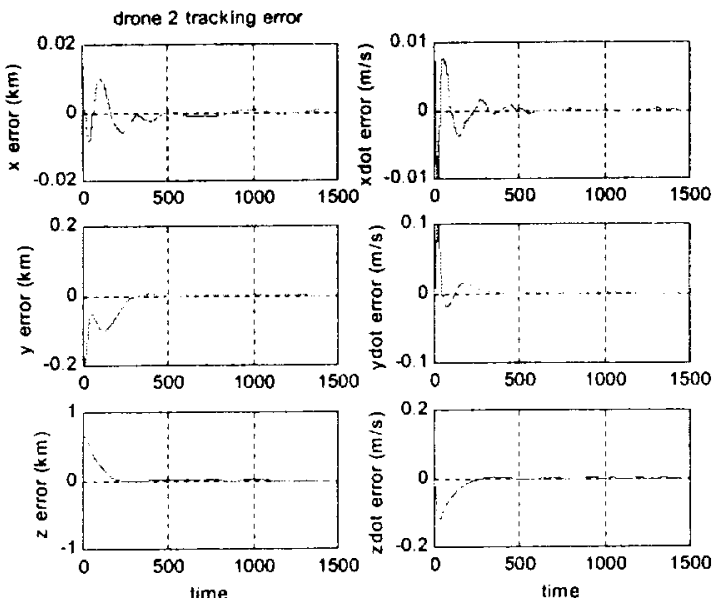

Figure 9. Drone 2 tracking error ( 90 degree slew and $0.5 \mathrm{~km}$ focal length)

Table 1 lists the total position tracking error after one day for the different scenarios when noise is turned off.

Table 1. Formation slewing position tracking errors with no noise

\begin{tabular}{|l|l|l|l|l|}
\hline $\begin{array}{c}\text { Focal } \\
\text { length } \\
(\mathrm{km})\end{array}$ & $\begin{array}{c}\text { Slew } \\
\text { angle } \\
(\mathrm{deg})\end{array}$ & $\begin{array}{c}\text { Hub } \\
\text { position } \\
\text { tracking } \\
\text { error }(\mathrm{m})\end{array}$ & $\begin{array}{c}\text { Drone2 } \\
\text { position } \\
\text { tracking } \\
\text { error }(\mathrm{m})\end{array}$ & $\begin{array}{c}\text { Drone 31 } \\
\text { position } \\
\text { tracking error } \\
(\mathrm{m})\end{array}$ \\
\hline 0.5 & 90 & $8.33 \mathrm{e}-6$ & $3.075 \mathrm{e}-6$ & $3.075 \mathrm{e}-6$ \\
\hline 0.5 & 30 & $3.05 \mathrm{e}-6$ & $1.126 \mathrm{e}-6$ & $1.126 \mathrm{e}-6$ \\
\hline 4 & 90 & $7.35 \mathrm{e}-5$ & $2.713 \mathrm{e}-5$ & $2.713 \mathrm{e}-5$ \\
\hline 4 & 30 & $2.69 \mathrm{e}-5$ & $9.93 \mathrm{e}-6$ & $9.93 \mathrm{e}-6$ \\
\hline
\end{tabular}

For all scenarios, neglecting noise, the velocity tracking error is essentially zero after one day. If noise is turned off, the tracking errors go asymptotically to zero, as expected with a linear quadratic regulator control strategy. Clearly, the noise and estimation errors have a significant effect on the tracking errors.

Figure 10 shows the estimation error results of a dozen simulations for the hub with varying slew angles and focal lengths. The red lines are the three-sigma values. The hub's steady-state position three-sigma values are about 50 meters, and the steady-state velocity three-sigma values are about 1 millimeter per second for all scenarios. The estimation errors are within the three-sigma values with few exceptions. The threesigma values change for each simulation (because the noise is random), but the change cannot be seen for the hub because the order of magnitude of change is much, much less than their overall value.

Figure 11 shows the estimation errors for a dozen simulations for the first drone satellite. Drone 2's estimation errors are, with few exceptions, within the three-sigma values for all scenarios.
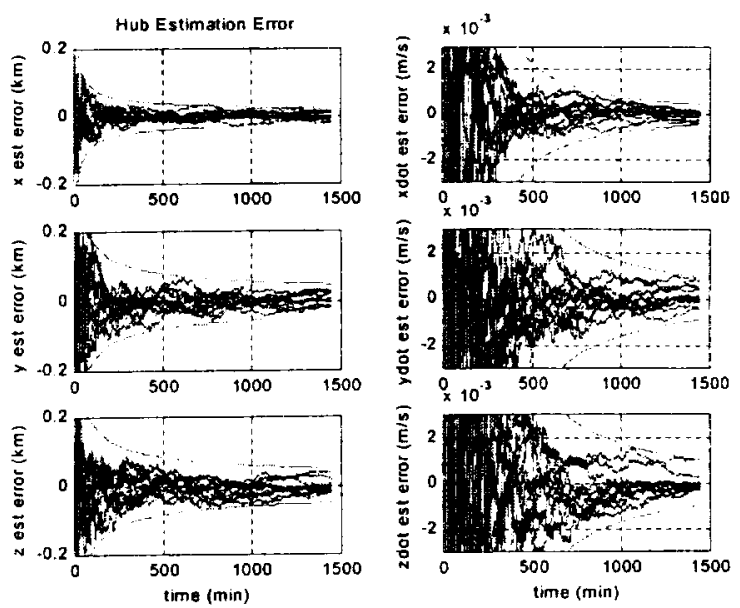

Figure 10. Hub estimation error for $0.5 \mathrm{~km}$ focal length and 90 degree slew
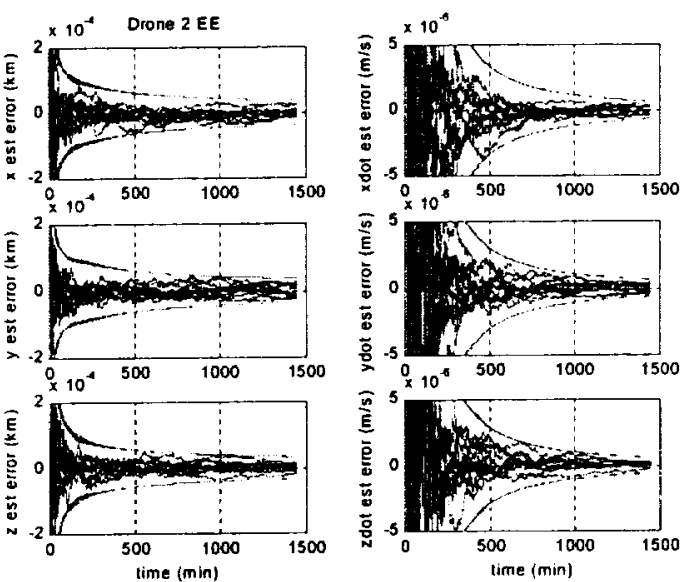

Figure 11. Drone 2 estimation errors for $0.5 \mathrm{~km}$ focal length and 90 degree slew

The three-sigma value change from one simulation to another can be seen in the drone estimation error plots. The range from the hub to the drone is either 0.5 or 4 $\mathrm{km}$, whereas the range from the hub to the earth is about 1.5 million $\mathrm{km}$.

From the control efforts, the directional $\Delta V$ can be determined as before but the time interval of 1440 minutes must be taken into account. The total $\Delta V$ is found from the directional $\Delta V$ 's. The differences between slewing $\Delta V \mathrm{~s}$ and orbit maintenance $\Delta V \mathrm{~s}$ are the number of maneuvers over the course of the simulation and the maneuver interval. The formation slewing simulation runs for one day, with one maneuver per minute (1440 maneuvers), whereas the Lissajous orbit simulation nuns for 359 days with one maneuver per day. Table 2 shows the average $\Delta V$ 's for a dozen simulations for the various scenarios. 
Table 2 Average formation slewing $\Delta V$ 's

\begin{tabular}{|l|l|l|l|l|}
\hline $\begin{array}{c}\text { Focal } \\
\text { Length } \\
(\mathrm{km})\end{array}$ & $\begin{array}{c}\text { Slew } \\
\text { Angle } \\
(\mathrm{deg})\end{array}$ & $\begin{array}{c}\text { Hub } \\
\Delta V_{(\mathrm{m} / \mathrm{s})}\end{array}$ & $\begin{array}{c}\text { Drone 2 } \\
\Delta V(\mathrm{~m} / \mathrm{s})\end{array}$ & $\begin{array}{c}\text { Drone 31 } \\
\Delta V^{(\mathrm{m} / \mathrm{s})}\end{array}$ \\
\hline 0.5 & 30 & 1.0705 & 0.8271 & 0.8307 \\
\hline 0.5 & 90 & 1.1355 & 0.9395 & 0.9587 \\
\hline 4 & 30 & 1.2688 & 1.1189 & 1.1315 \\
\hline 4 & 90 & 1.8570 & 2.1907 & 2.1932 \\
\hline
\end{tabular}

The larger the angle the formation slews through, the more $\Delta V$ is needed. Also, the larger the focal length, the more $\Delta V$ required. Table 3 shows the corresponding propellant masses needed to achieve the $\Delta V$ 's given in Table 2 , with the assumptions that the Isp of the low-thrust thrusters is 10000 seconds, the initial mass of the hub is $550 \mathrm{~kg}$, and the initial mass of each drone is $100 \mathrm{~kg}$.

Table 3. Average formation slewing propellant masses

\begin{tabular}{|c|c|c|c|c|}
\hline $\begin{array}{c}\text { Focal } \\
\text { Length } \\
(\mathrm{km})\end{array}$ & $\begin{array}{l}\text { Slew } \\
\text { Angle } \\
\text { (deg) }\end{array}$ & $m_{\text {prop }}^{\text {Hub }}$ & $\begin{array}{l}\text { Drone } 2 \\
m_{\text {prop }}(\mathrm{g})\end{array}$ & $\begin{array}{l}\text { Drone } 31 \\
m_{\text {prop }}(\mathrm{g})\end{array}$ \\
\hline 0.5 & 30 & 6.0018 & 0.8431 & 0.8468 \\
\hline 0.5 & 90 & 6.3662 & 0.9577 & 0.9773 \\
\hline 4 & 30 & 7.1135 & 1.1406 & 1.1534 \\
\hline 4 & 90 & 10.4112 & 2.2331 & 2.2357 \\
\hline
\end{tabular}

When the noise is turned off, the required $\Delta V$ and propellant mass is reduced significantly. Table 4 shows the $\Delta V$ 's and Table 5 shows the corresponding propellant masses when noise is removed.

Table 4. Required $\Delta V$ 's for formation slewing cases without noise

\begin{tabular}{|c|c|c|c|c|}
\hline $\begin{array}{c}\text { Focal } \\
\text { Length } \\
(\mathrm{km})\end{array}$ & $\begin{array}{c}\text { Slew } \\
\text { Angle } \\
(\mathrm{deg})\end{array}$ & $\begin{array}{c}\text { Hub } \\
\Delta V(\mathrm{~m} / \mathrm{s})\end{array}$ & $\begin{array}{c}\text { Drone 2 } \\
\Delta V(\mathrm{~m} / \mathrm{s})\end{array}$ & $\begin{array}{c}\text { Drone 31 } \\
\Delta V(\mathrm{~m} / \mathrm{s})\end{array}$ \\
\hline 0.5 & 30 & 0.0504 & 0.0853 & 0.0998 \\
\hline 0.5 & 90 & 0.1581 & 0.2150 & 0.2315 \\
\hline 4 & 30 & 0.4420 & 0.5896 & 0.6441 \\
\hline 4 & 90 & 1.3945 & 1.9446 & 1.9469 \\
\hline
\end{tabular}

Table 5. Required propellant masses for formation slewing cases without noise

\begin{tabular}{|c|c|c|c|c|}
\hline $\begin{array}{c}\text { Focal } \\
\text { Length } \\
(\mathrm{km})\end{array}$ & $\begin{array}{c}\text { Slew } \\
\text { Angle } \\
\text { (deg) }\end{array}$ & $\begin{array}{c}\text { Hub } \\
m_{\text {prop }}(\mathrm{g})\end{array}$ & $\begin{array}{c}\text { Drone 2 } \\
m_{\text {prop }}(\mathrm{g})\end{array}$ & $\begin{array}{c}\text { Drone 31 } \\
m_{\text {prop }}(\mathrm{g})\end{array}$ \\
\hline 0.5 & 30 & 0.2826 & 0.0870 & 0.1017 \\
\hline 0.5 & 90 & 0.8864 & 0.2192 & 0.2360 \\
\hline 4 & 30 & 2.4781 & 0.6010 & 0.6566 \\
\hline 4 & 90 & 7.8182 & 1.9822 & 1.9846 \\
\hline
\end{tabular}

\section{Formation Reorientation}

For some stars, one snapshot from the SI formation will not provide enough sampling data for sufficient resolution. In these cases, the drones must rotate 90 degrees and take another snapshot. The aim from the drones, through the hub, to the desired star, is maintained with such a reorientation. Figure 12 shows the first four drones of the formation before and after reorientation (with a $0.5 \mathrm{~km}$ focal length). Only four drones are pictured for clarity.
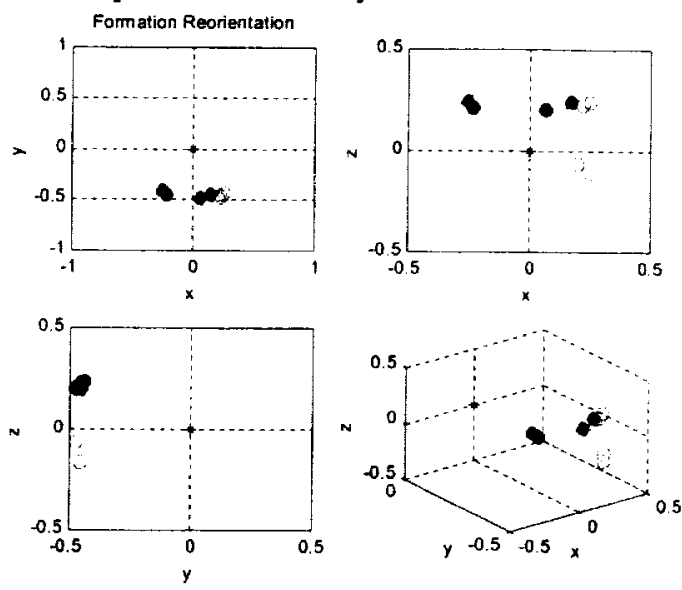

Figure 12. Formation reorientation $(0.5 \mathrm{~km}$ focal length)

The scale is in kilometers in all four pictures. The plot in the upper-right shows the projection of the formation in the $x-z$ plane. The black dots are the drones at their initial conditions, and the open circles are the drones after the simulation. The formation appears to have rotated clockwise 90 degrees about the $y$ axis, but the hub is into the page, so the rotation is counterclockwise when viewed from the hub. Figures 13 and 14 show the tracking errors for the hub and the drone for the $0.5 \mathrm{~km}$ focal length case.
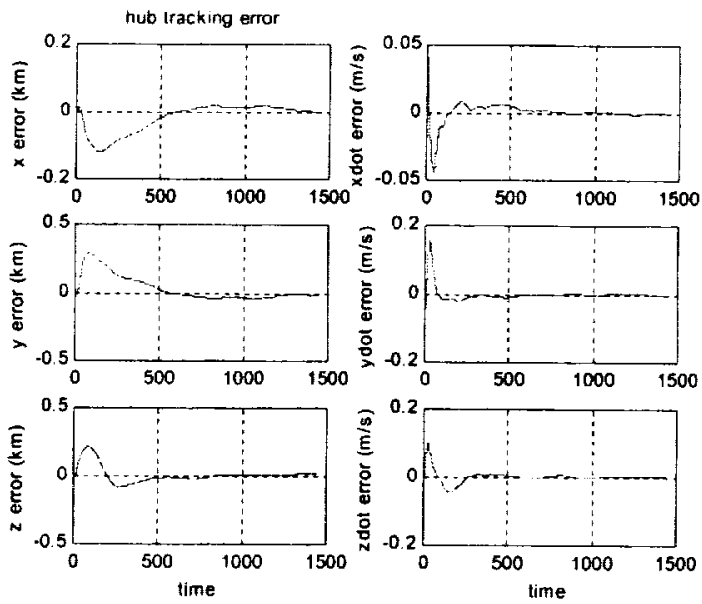

Figure 13 . Hub tracking error ( $0.5 \mathrm{~km}$ focal length)

The hub tracks to within 40 meters of its reference position, and to within 8 millimeters per second of its reference velocity for both focal lengths. 

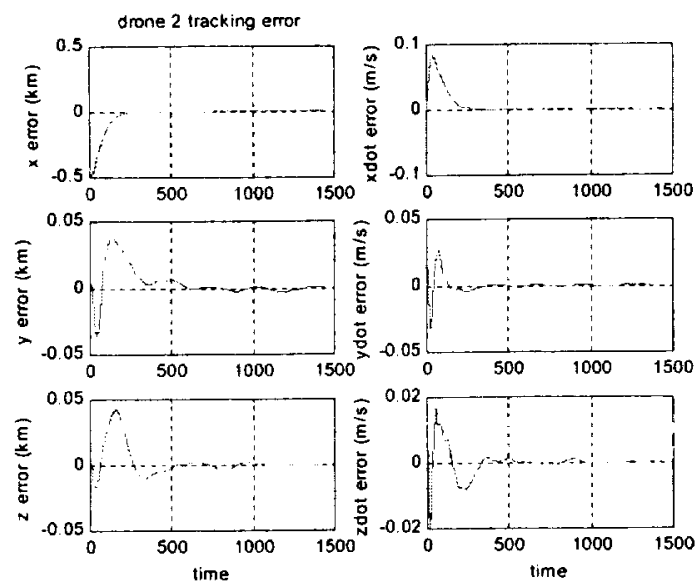

Figure 14. Drone 2 tracking error $(0.5 \mathrm{~km}$ focal length)

Every drone tracks to within 4 meters of its reference position, and to within 1.5 millimeters per second of its desired zero velocity for both focal lengths.

When noise is turned off, the satellites track much better than when the noise is included. Table 6 shows the total position tracking errors for the various satellites after one day, when noise is eliminated.

Table 6. Formation reorientation position tracking errors with no noise

\begin{tabular}{|c|c|c|c|}
\hline $\begin{array}{c}\text { Focal length } \\
(\mathrm{km})\end{array}$ & $\begin{array}{c}\text { Hub position } \\
\text { tracking } \\
\text { error }(\mathrm{m})\end{array}$ & $\begin{array}{c}\text { Drone 2 } \\
\text { position } \\
\text { tracking } \\
\text { error }(\mathrm{m})\end{array}$ & $\begin{array}{c}\text { Drone 31 } \\
\text { position } \\
\text { tracking error } \\
(\mathrm{m})\end{array}$ \\
\hline 0.5 & $2.147 \mathrm{e}-6$ & $0.792 \mathrm{e}-6$ & $0.793 \mathrm{e}-6$ \\
\hline 4 & $2.146 \mathrm{e}-6$ & $0.792 \mathrm{e}-6$ & $0.793 \mathrm{e}-6$ \\
\hline
\end{tabular}

The tracking errors go asymptotically to zero, and the velocity tracking errors are essentially zero at the end of a day. Just as with the formation slewing simulation, the noise, and in turn the estimation errors, are the largest reason for imperfect tracking.

Since the hub and drone estimation errors for a dozen simulations with the different focal lengths is similar to the slew simulations, they are not shown here. The steady-state $x$ three-sigma value is about 30 meters. The steady-state $y$ and $z$ three-sigma value is about 50 meters. The steady-state velocity three-sigma values are about 1 millimeter per second. The hub estimation errors stay within the three-sigma values except for rare occasions. For any drone and either focal length, the steady-state position three-sigma values are less than 0.1 meters, and the steady-state velocity threesigma values are less than 1e-6 meters per second. Also, the estimation errors stay within the three-sigma values with rare exceptions.

The $\Delta V$ can be determined, for each satellite, from the control effort required to reorient the forma- tion. Table 7 gives the average $\Delta V$ 's from a dozen simulations to reorient the formation.

Table 7. Average formation reorientation $\Delta V$ 's

\begin{tabular}{|c|c|c|c|}
\hline $\begin{array}{c}\text { Focal Length } \\
(\mathrm{km})\end{array}$ & $\begin{array}{c}\text { Hub } \\
\Delta V(\mathrm{~m} / \mathrm{s})\end{array}$ & $\begin{array}{c}\text { Drone 2 } \\
\Delta V(\mathrm{~m} / \mathrm{s})\end{array}$ & $\begin{array}{c}\text { Drone 31 } \\
\Delta V(\mathrm{~m} / \mathrm{s})\end{array}$ \\
\hline 0.5 & 1.0126 & 0.8421 & 0.8095 \\
\hline 4 & 1.0133 & 0.8496 & 0.8190 \\
\hline
\end{tabular}

The focal length has no discernible effect on the $\Delta V$ needed to reorient the formation. This makes sense because the rotation is about the $y$-axis, and the focal length is assumed to be the measurement along the $y$ axis from the hub to the drones. Table 8 gives the propellant masses that correspond to Table 7 .

Table 8. Average formation reorientation propellant

\begin{tabular}{|c|c|c|c|}
\hline $\begin{array}{c}\text { Focal Length } \\
(\mathrm{km})\end{array}$ & $\begin{array}{c}\text { Hub } \\
m_{\text {prop }}(\mathrm{g})\end{array}$ & $\begin{array}{c}\text { Drone } 2 \\
m_{\text {prop }}(\mathrm{g})\end{array}$ & $\begin{array}{c}\text { Drone 31 } \\
m_{\text {prop }}(\mathrm{g})\end{array}$ \\
\hline 0.5 & 5.6771 & 0.8584 & 0.8252 \\
\hline 4 & 5.6811 & 0.8661 & 0.8349 \\
\hline
\end{tabular}

Without noise, the $\Delta V$ needed to reorient the formation is much less, as shown in Table 9.

Table 9. Required $\Delta V$ for formation reorientation without noise

\begin{tabular}{|c|c|c|c|}
\hline $\begin{array}{c}\text { Focal Length } \\
(\mathrm{km})\end{array}$ & $\begin{array}{c}\text { Hub } \\
\Delta V(\mathrm{~m} / \mathrm{s})\end{array}$ & $\begin{array}{c}\text { Drone 2 } \\
\Delta V(\mathrm{~m} / \mathrm{s})\end{array}$ & $\begin{array}{c}\text { Drone 31 } \\
\Delta V(\mathrm{~m} / \mathrm{s})\end{array}$ \\
\hline 0.5 & 0.0408 & 0.1529 & 0.1496 \\
\hline 4 & 0.0408 & 0.1529 & 0.1495 \\
\hline
\end{tabular}

Table 10 gives the propellant masses that correspond to Table 9 .

Table 10. Required propellant masses for formation reorientation without noise

\begin{tabular}{|c|c|c|c|}
\hline $\begin{array}{c}\text { Focal Length } \\
(\mathrm{km})\end{array}$ & $\begin{array}{c}\text { Hub } \\
m_{\text {prop }}(\mathrm{g})\end{array}$ & $\begin{array}{c}\text { Drone 2 } \\
m_{\text {prop }}(\mathrm{g})\end{array}$ & $\begin{array}{c}\text { Drone 31 } \\
m_{\text {prop }}(\mathrm{g})\end{array}$ \\
\hline 0.5 & 0.2287 & 0.1623 & 0.1525 \\
\hline 4 & 0.2287 & 0.1623 & 0.1524 \\
\hline
\end{tabular}

\section{SUMMARY AND CONCLUSION}

The control strategy and Kalman filter provided satisfactory results. The hub satellite tracks to its reference orbit sufficiently for the SI mission requirements. The drone satellites, on the other hand, track to only within a few meters. Without noise, though, the drones track to within several micrometers. The first tier of the proposed control scheme for SI requires the drones to track within centimeters. This could be accomplished with better sensors to lessen the effect of the process and measurement noise. Tuning the con- 
troller and varying the maneuver intervals should provide additional savings as well. The Kalman filter performed such that the estimation errors were for the most part within the three-sigma values. The propellant mass and $\Delta V$ results provide a minimum design boundary for the SI mission. Additional propellant will be needed to perform all attitude maneuvers, tighter control requirement adjustments, and other mission functions.

Future studies must examine the attitude dynamics and control problem and integrate this into the translation control. Other items that should be considered in the future include; non-ideal thrusters, collision avoidance, system reliability and fault detection, and nonlinear control and estimation. For the nanometer level preciseness of the second and third control tiers, new control strategies and algorithms may be required.

\section{REFERENCES}

1. "Magnetospheric Constellation," http://stp.gsfc.nasa.govimissions/mcimc.htm. October 2001

2. "The GSFC Stellar Imager Homepage," http:/hires.gsfc.nasa.govi-si . October, 2001.

3. "Micro Arcsecond X-Ray Imaging Mission," http://maxim.gsfc.nasa.gov. October, 2001

4. "Constellation-X", http:/iconstellationX/docs ga genaudience.html, May 2001

5. "Laser Interferometer Space Antenna," http://lisa.jpl.nasa.gov. October 2001..

6. Farquhar, R., The Control and Use of LibrationPoint Satellites. NASA Technical Report R-346. 1970.

7. Gomez, G., Masdemont, J., and Simo, C., "Quasihalo Orbits Associated with Libration Points," Journal of the Astronautical Sciences, Vol. 46. 1998.

8. Scheeres, D.J., and Vinh, N.X., "Dynamics and Control of Relative Motion in an Unstable Orbit," AIAA Paper 2000-4135. 2000.

9. Hoffman, D.A., Station-keeping at the Collinear Equilibrium Points of the Earth-Moon System, JSC-26189. NASA Johnson Space Center. 1993.

10. Wie, B., Space Vehicle Dynamics and Control. AIAA Education Series. 1998.

11. Carpenter, K.G., Neff, S.G., Schrijver, C.J., Allen, R.J., and Rajagopal, J., "The Stellar Imager (SI) Mission Concept." In proceedings of the $36^{\text {th }}$ Liege Astrophysical Colloquium: From Optical to Millimetric Intefereometry: Scientific and Technical Challenges. 2001.

12. Leitner, J., and Schnurr, R., "Stellar Imager (SI): Formation Flying." Presentation from the Integrated Mission Design Center, Goddard Space Flight Center, NASA. 2001.
13. Howell, K.C., and Anderson, J., Generator User's Guide. Version 3.0.2. 2001.

14. Brown, R.C., and Hwang, P.Y.C., Introduction to Random Signals and Applied Kalman Filtering. $2^{\text {nd }}$ Edition. John Wiley \& Sons, Inc. 1992.

15. Golomb, S.W., and Taylor, H. IEEE Trans. Info. Theo., 28, \#4. 1982.

\section{Appendix: SI Formation Geometry}

The hub spacecraft is assumed to be at the center of a sphere, on which the drones lie. The $x \mathrm{di}$ rection of the hub-centered Cartesian coordinate system is parallel to and in the same direction as the radial axis of the earth-centered rotating coordinate system used by Generator. The $y$ direction of the hub-centered system is parallel to and in the same direction as the alongtrack direction of the earth-centered system. The $z$ direction of the hub-centered system is parallel to and in the same direction as the cross-track direction of the earth-centered system. Figure 14 shows the relationship between the two coordinate systems.

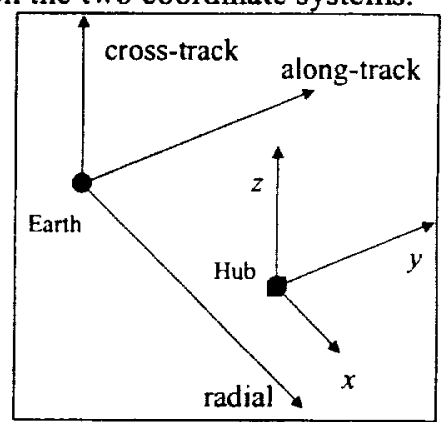

Figure 14. Hub-centered Cartesian coordinate system

The drones' positions are expressed in the spherical coordinates, $\mathrm{r}, \theta$, and $\phi$, which relate to the hubcentered Cartesian coordinates by

$$
x=r \sin (\theta), \quad y=r \cos (\theta) \sin (\phi) \text {, }
$$$$
\text { and } z=r \cos (\theta) \cos (\phi)
$$

The radial coordinate, $r$, is the distance from the hub to the drone and always equals the radius of the sphere, which is twice the desired focal length. The $\phi$ coordinate is measured from the positive $z$ axis toward the positive $y$ axis. Standing on the positive $x$ axis and looking back, a positive rotation is clockwise. The $\theta$ coordinate is measured from the position in the $y-z$ plane along the sphere in a clockwise direction when considered from the positive $\mathrm{z}$ axis looking back.

Initially, the center of the formation will be placed directly behind the hub in the along-track direction. Thus, 


$$
\theta_{c e n t}^{0}=0 \text { and } \phi_{c e n t}^{0}=\frac{3 \pi}{2}
$$

where $\theta_{\text {cent }}^{0}$ and $\phi_{c e n t}^{0}$ are initial coordinates corresponding to an imaginary central drone satellite. There are 30 drones for the SI mission, so 15 have a $\phi^{0}$ coordinate greater than $\phi_{\text {cent }}^{0}$, and 15 have a $\phi^{0}$ coordinate less than $\phi_{\text {cent }}^{0}$. Knowing that the maximum distance between drones is $0.5 \mathrm{~km}$, or $0.25 \mathrm{~km}$ to the imaginary central drone, the coordinates can be calculated by

$$
\phi_{i}^{0}=\phi_{c c t}^{0}+\frac{17-i}{15} \sin ^{-1}\left(\frac{0.25}{r}\right),
$$

where $i$ represents the $2^{\text {nd }}$ through $16^{\text {th }}$ satellite (the hub being number 1 ), and

$$
\phi_{i}^{0}=\phi_{c e n t}^{0}-\frac{i-16}{15} \sin ^{-1}\left(\frac{0.25}{r}\right),
$$

where i represents the $17^{\text {th }}$ through $31^{\text {st }}$ satellite. The $\theta^{\circ}$ coordinates can be determined in a similar fashion, for i from 2 to 31 ,

$$
\theta_{i}^{0}=\theta_{c e n t}^{0}+\frac{j}{15} \sin ^{-1}\left(\frac{0.25}{r}\right)
$$

where $j$ is an integer between -15 and 15 , such that each satellite, $i$, has a unique $j$. This formation is a Golomb $^{31}$ rectangle laid out on a spherical surface rather than a plane. The spherical coordinates for each drone and the imaginary central drone are intermediately transformed to Cartesian coordinates yielding ${ }^{\text {int }} x_{i}^{0},{ }^{\text {int }} y_{i}^{0},{ }^{\text {int }} z_{i}^{0}, x_{c e n t}^{0}, y_{c e n t}^{0}$, and $z_{c e n t}^{0}$. Finally, the satellites are translated by half of the imaginary central coordinates to account for the hub being halfway between the origin of the sphere and the surface of the sphere:

$$
\begin{gathered}
x_{i}^{0}={ }^{\mathrm{int}} x_{i}^{0}-\frac{x_{c e n t}^{0}}{2}, y_{i}^{0}={ }^{\mathrm{int}} y_{i}^{0}-\frac{y_{c e n t}^{0}}{2}, \\
\text { and } z_{i}^{0}={ }^{\mathrm{int}} z_{i}^{0}-\frac{z_{c e n t}^{0}}{2}
\end{gathered}
$$

The drones initially start with zero relative velocity to the hub, so for $\mathrm{i}$ from 2 to 31 ,

$$
\dot{x}_{i}^{0}=\dot{y}_{i}^{0}=\dot{z}_{i}^{0}=0,
$$

Two types of maneuvers are considered in this paper; slews and reorientations. In a slew, the reference states are built by rotating the formation through a slew angle, $\alpha$. The rotation is about the hub-centered $\mathrm{x}$ axis. Thus, for $i$ from 2 to 31 ,

$$
\begin{aligned}
& \phi_{i}^{r e f}=\phi_{i}^{0}-\alpha, \quad \theta_{i}^{r e f}=\theta_{i}^{0} \\
& , \phi_{c e n t}^{r e f}=\phi_{c e n t}^{0}-\alpha, \theta_{c e n t}^{r e f}=\theta_{c e n t}^{0}=0
\end{aligned}
$$

These spherical reference coordinates used to yield the intermediate ${ }^{\text {int }} x_{i}^{\text {ref }},{ }^{\text {int }} y_{i}^{\text {ref }},{ }^{\text {int }} z_{i}^{\text {ref }}, x_{\text {cent }}^{\text {ref }}, y_{\text {cent }}^{\text {ref }}$, and $z_{\text {cent }}^{r e f}$. The drones must be translated by half of the imaginary central drone position:

$$
\begin{aligned}
& x_{i}^{r e f}={ }^{i n t} x_{i}^{r e f}-\frac{x_{c e n t}^{r e f}}{2} \\
& y_{i}^{r e f}={ }^{i n t} y_{i}^{r e f}-\frac{y_{c e n t}^{r e f}}{2} \\
& z_{i}^{r e f}={ }^{\text {int }} z_{i}^{r e f}-\frac{z_{c e n t}^{r e f}}{2} .
\end{aligned}
$$

No relative velocities between the hub and drones are desired, so for i from 2 to 31 ,

$$
\dot{x}_{i}^{\text {ref }}=\dot{y}_{i}^{\text {ref }}=\dot{z}_{i}^{\text {ref }}=0 \text {. }
$$

The drones' reference states do not change over the course of the simulation.

The hub satellite's initial and reference conditions are treated differently than the drones', because the hub's reference states change over the duration of the simulation. From Generator, the hub reference conditions are known at the beginning of the simulation, $\mathbf{x}_{1}^{\text {refo }}$, and at the end of simulation, one day later, $\mathbf{x}_{1}^{\text {refend }}$. The hub reference states at some epoch, $k$, during the simulation are determined by linear interpolation,

$$
\mathbf{x}_{1}^{\text {refk }}=\left(\mathbf{x}_{1}^{\text {refend }}-\mathbf{x}_{1}^{\text {ref } 0}\right) \frac{d T}{1440} k+\mathbf{x}_{1}^{\text {ref } 0} .
$$

The development for the formation reorientation is nearly identical to the formation slewing simulation. The difference between the formation reorientation and formation slewing simulations is the desired reference states of the drones. The formation slewing reference states are based on a variable slew angle, $\alpha$, rotation about the $\mathrm{x}$ axis, whereas the formation reorientation reference states are based on a 90 degree rotation about the $y$ axis. The imaginary central drone has the same reference conditions as initial conditions,

$\phi_{c e n t}^{r e f}=\phi_{c e n t}^{0}=\frac{3 \pi}{2}, \theta_{c e n t}^{r e f}=\theta_{c e n t}^{0}=0$

Setting

$\phi_{i}^{r e f}=-\theta_{i}^{0}+\phi_{c e n t}^{0}, \theta_{i}^{r e f}=\phi_{i}^{0}-\phi_{c e n t}^{0}$

for $\mathrm{i}$ from 2 to 31 , gives the drones' reference positions. The rotation is 90 degrees counterclockwise about the $y$ axis when looking back from the hub. Once again, zero relative velocity between the drones and the hub is desired. 
